\title{
Patterning instability on the Mars polar ice caps
}

\author{
Felix S. L. $\mathrm{Ng}^{1,2}$ and Maria T. Zuber ${ }^{1}$ \\ Received 13 July 2005; revised 4 November 2005; accepted 10 November 2005; published 18 February 2006.
}

[1] We present a mathematical theory to study the origin of large-scale spiral troughs on the Mars residual polar caps, starting with the hypothesis that atmospheric circulation governs the planform of the troughs via an instability that operates in the flow direction of surface winds. This concept can explain why the troughs spiral at each pole in an opposite sense to that expected for Coriolis-deflected winds. The instability arises from interactions on water ice, assumed to contain dust, and depends on how the exchange of atmospheric dust and moisture $\left(\mathrm{H}_{2} \mathrm{O}\right)$ with the polar cap surface controls its albedo and mass and energy balance. Our model predicts spatial patterns to form when moisture is carried by wind over the surface, owing to unstable coupling between the albedo and the $\mathrm{H}_{2} \mathrm{O}$ vapor pressure. The resulting albedo pattern causes an alternating "accumulation-ablation" mass balance, so that an undulating topography develops which resembles the (dark) troughs and their adjacent (bright) smooth terrains on the polar caps. Because the albedo patterning process is fast, whereas topographic evolution is slow, we suggest that an ancient imprint in the surface albedo preconditions today's trough morphology.

Citation: Ng, F. S. L., and M. T. Zuber (2006), Patterning instability on the Mars polar ice caps, J. Geophys. Res., 111, E02005, doi:10.1029/2005JE002533.

\section{Introduction}

[2] The spiral troughs on the polar residual ice caps of Mars (Figure 1a) have no terrestrial analogue [Thomas et al., 1992], attesting the special conditions under which surface processes shape ice and other deposits on that planet. This paper focuses on the mechanisms of trough formation; it tries to model the underlying volatile and dust physics, motivated by the view that such physics are crucial for deciphering climate information from the deposits, as well as their geologic history. The troughs have a prominent topographic expression (Figure 1b) [Zuber et al., 1998; Smith et al., 2001a]. Early in the summer on each ice cap, they become strikingly visible as a meter-thick frost of $\mathrm{CO}_{2}$ retreats [Smith et al., 2001b]. Sublimation of this seasonal veneer exposes the dusty surfaces of the troughs and their equator-facing scarps, which have much lower albedo than the intervening ice-rich smooth terrains (Figure 1c). The reason for the troughs to spiral is unknown, although studies of Viking imagery interpret them as ablation features [Blasius et al., 1982; Howard et al., 1982], which is consistent with later observations that the trough cross section is asymmetric [Smith et al., 1998; Zuber et al., 1998]. The early studies [Blasius et al., 1982; Howard et al., 1982] also suggest that troughs may migrate poleward by "accublation" sublimation of ice at the troughs and at the scarps, and deposition of snow or ice on the smooth terrains

\footnotetext{
${ }^{1}$ Department of Earth, Atmospheric and Planetary Sciences, Massachusetts Institute of Technology, Cambridge, Massachusetts, USA.

${ }^{2}$ Now at Department of Geography, University of Sheffield, Sheffield, UK.

Copyright 2006 by the American Geophysical Union. 0148-0227/06/2005JE002533\$09.00
}

[Howard, 1978; Fisher, 1993, 2000]. Such a mass balance configuration may develop because surface slope controls insolation received by the ice. Whether or not this idea is correct, the slow migration rate of the troughs has not been measured.

[3] How did the troughs initiate? Had they formed repeatedly at the ice margin, the interplay of migration and timing may suffice to explain their spacing (tens of kilometers) and orientation. To model this interplay, however, is difficult because the margin boundary conditions are uncertain. An idea by Howard [1978] that sun-angle bias causes scarps to migrate into a spiral planform also remains tentative. Alternatively, new troughs could form on the ice. We explore this explanation [see $\mathrm{Ng}$ and Zuber, 2003] and describe an instability mechanism whereby a featureless ice cap becomes patterned in the surface albedo spontaneously; in turn, this induces an accublation mass balance, such that undulations develop on the cap surface, and they grow into troughs.

[4] Pelletier [2004] numerically simulated two-dimensional features that mimic the spiral troughs by fueling instability of the ice surface. However, his model lacks strong physical basis, making it difficult to evaluate what his instability mechanism involves in reality. Also, like earlier theories of the troughs, his treatment of the surface processes ignores the atmosphere, particularly how moisture $\left(\mathrm{H}_{2} \mathrm{O}\right)$ and wind-blown dust over the ice regulate its albedo and mass and energy fluxes. That these interactions are important is clear from Viking observations of the summer north polar cap [Farmer et al., 1976; Kieffer et al., 1976; Bass and Paige, 2000] showing that the surface albedo, surface temperature, and atmospheric water abundance are coupled. Our model below shows that this coupling can become spatially unstable. In the corresponding trough- 

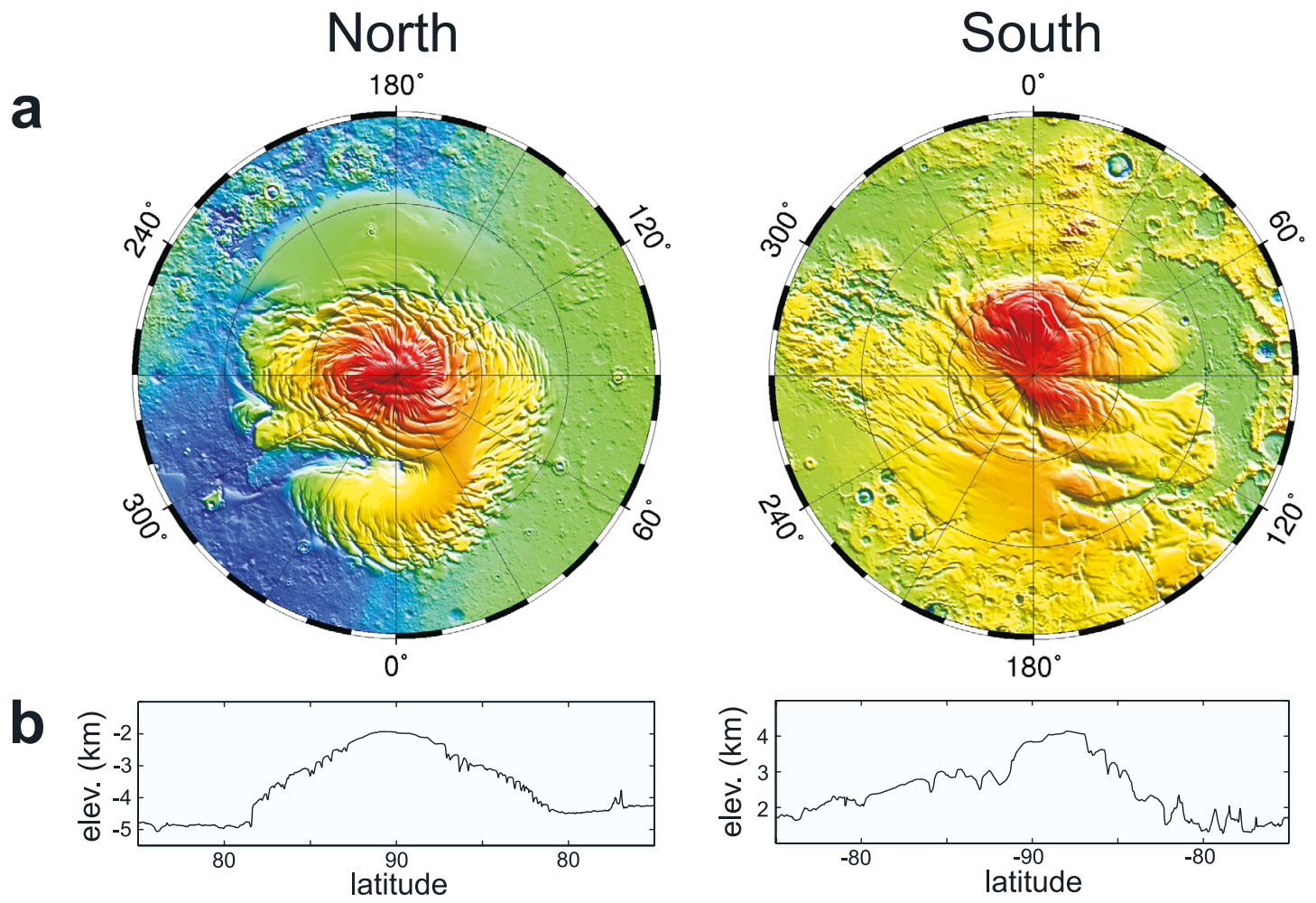

C
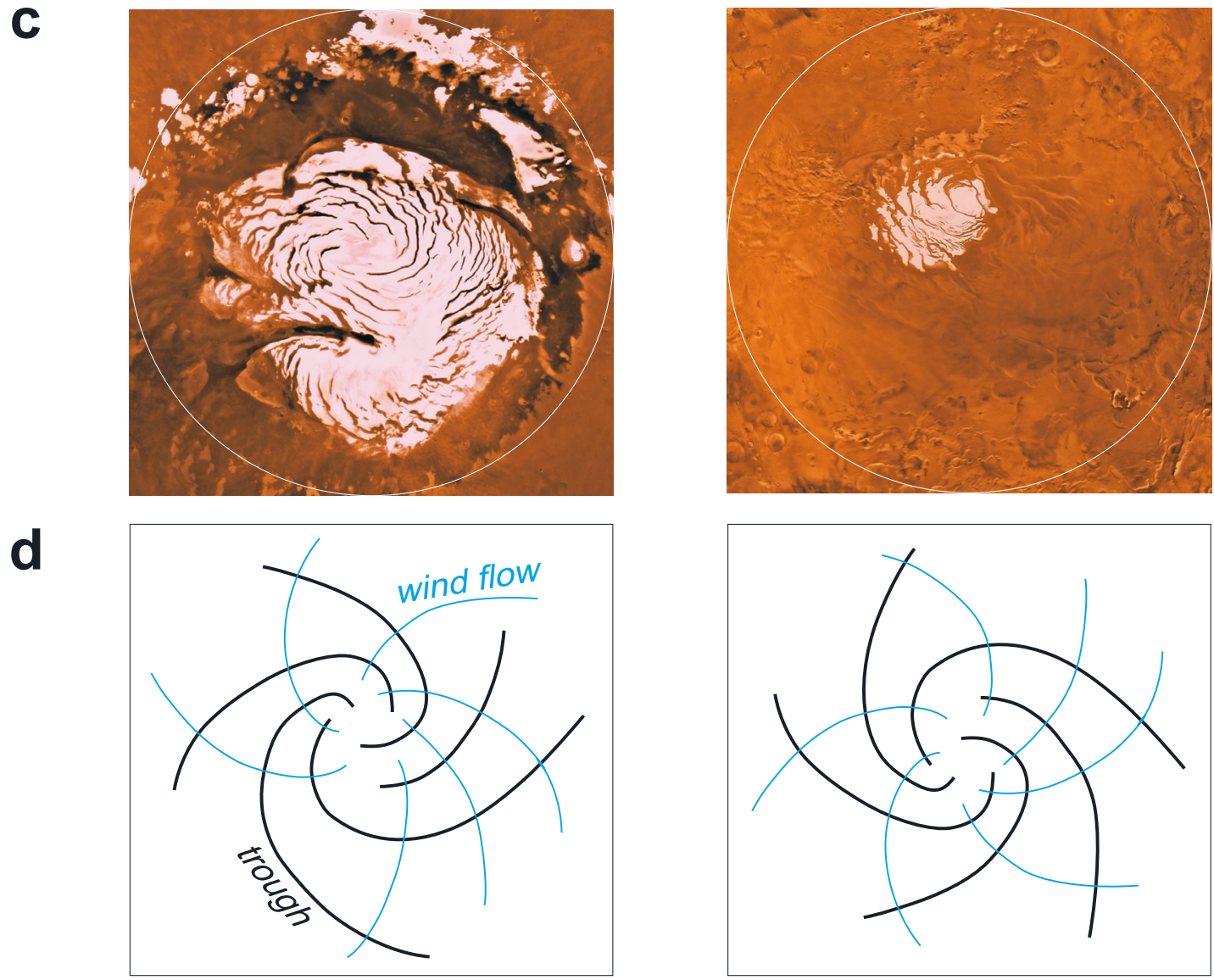

Figure 1 


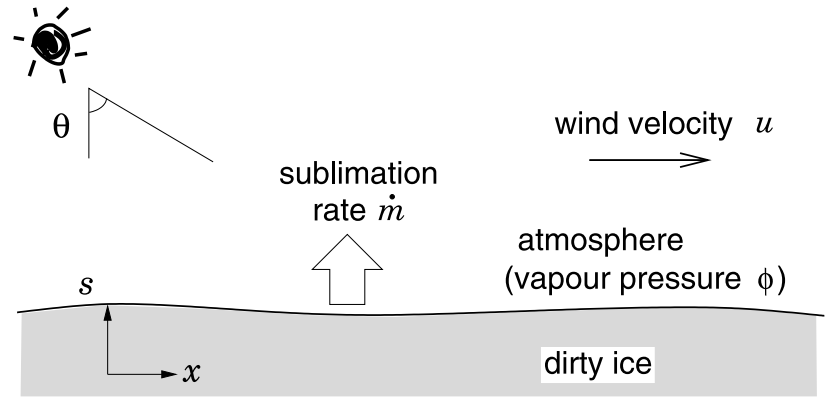

Figure 2. Cartoon of the polar cap surface and definition of several key variables in our model.

forming mechanism, a key feedback destabilizes the surface albedo, not topography, and trough migration assumes secondary importance. Thus although the surface evolution depends on slope, that is not why instability occurs.

\section{Coriolis Origin of Spiraling}

[5] We begin by assuming that the trough planform is governed by atmospheric circulation, but not as a result of wind-driven (mechanical) erosion of ice. A geometrical conundrum motivates this idea. Figure 1 shows that the northern troughs spiral out anticlockwise whereas the southern troughs spiral out clockwise, albeit less conspicuously. However, under Coriolis forces, surface winds at each pole should spiral the other way, which is inconsistent with an erosion origin of the troughs. Cutts [1973] had observed Coriolis deflection of linear markings outside the southern cap (on the layered deposits between $\sim 60^{\circ} \mathrm{S}$ and $75^{\circ} \mathrm{S}$ ) and argued that they have been sculpted by off-pole katabatic winds. Notice that based on dynamical consideration, the sense of spiraling of the surface wind pattern expected at each pole is the same whether the winds blow poleward or equatorward (e.g., winds in the Northern Hemisphere blow anticlockwise into a surface low and clockwise out of a surface high [Houghton, 1986]). But the cooling effect of ice means that katabatic winds associated with high pressure are likely to prevail in the polar summer; this has recently been demonstrated by numerical simulations of the north polar atmosphere [Tyler and Barnes, 2005].

[6] If we suppose that the troughs tend to be oriented perpendicularly to the surface winds, the conundrum may be resolved, for such geometry allows counter spiraling of the trough and wind systems (Figure 1d). This hypothesis requires an instability to form multiple troughs along wind, and our purpose is to model this behavior.

[7] Two observations support the hypothesis. First, the trough systems spiral in opposite senses in the north and south, consistent with the opposite polar rotations. This outcome would have to be a coincidence in Pelletier's [2004] model, where Coriolis effects are absent, and the simulated troughs may spiral either way depending on the initial conditions chosen. Second, high-resolution images of the north summer cap reveal "frost streaks" near the troughs and at high angle to their axes [Howard, 2000]. Interpreted to be eolian in origin, these features provide strong evidence for a Coriolis-deflected wind pattern, even though it is ambiguous which end of a frost streak points in the wind direction.

[8] Spectral measurements have long ago established a water-ice composition for the northern cap [Kieffer et al., 1976] and recently suggested the same for the southern cap [Bibring et al., 2004], whose relatively high crater density, however, reflects resurfacing rates that are slow compared to the north [Herkenhoff and Plaut, 2000]. The southern cap surface has been regarded as unusual for incomplete $\mathrm{CO}_{2}$ defrosting there during summer [Byrne and Ingersoll, 2003] and a scarp morphology distinct from that in the north $[\mathrm{Kolb}$ and Tanaka, 2001; Byrne and Ivanov, 2004]. The southern spiral trough system is also more irregular in plan view than the northern one. Despite these differences, we use a general formulation here to address interactions that may have occurred on both polar caps.

\section{Mathematical Model}

[9] We propose a model (see Figure 2) of how the surface elevation of an ice cap, $s$, varies with poleward distance, $x$, on a given wind trajectory (without fixing which way wind blows for the moment), and write $s=s(x, t)$ where $t$ denotes time. We assume water ice, and consider strictly the Mars summer season when the residual ice caps are exposed to the atmosphere. Dependent variables in our equations refer to summer averages, so only interactions at annual or longer timescales are resolved. To make our model one dimensional, we lump the summer insolation by using a constant zenith angle over time, $\theta$, although $\theta(x)$ may be prescribed to represent complex geometries arising from latitude and the wind's spiral trajectory. Corrections for crosswind slope, wind convergence, and Mars orbit variations, which require precise specification of the wind field (Figure 1d is schematic only), are ignored.

Figure 1. Large-scale spiral troughs on the (left) north and (right) south polar ice caps on Mars and our hypothesis of their relation with Coriolis-deflected polar winds. (a) Color-shaded surface elevations from the Mars Orbiter Laser Altimeter (MOLA) from pole to $75^{\circ}$ latitude. (Radial patterns at image centers are artifacts due to sparse data coverage of the poles by MOLA.) On Mars, $1^{\circ}$ of latitude corresponds to approximately $59 \mathrm{~km}$ in distance. (b) MOLA cross sections $\left(270^{\circ} \mathrm{E}-90^{\circ} \mathrm{E}\right.$ in left plot, $220^{\circ} \mathrm{E}-40^{\circ} \mathrm{E}$ in right plot) showing surface topography consisting of troughs and equator-facing scarps, separated by smooth terrains. Vertical exaggeration is $\approx 100: 1$. (c) Mars digitized image mosaics from the Viking mission showing spiral patterns in the surface albedo. The bright central areas are the residual caps; they and the surrounding areas are collectively called polar-layered deposits. Thin white circles mark the $75^{\circ}$ latitude. (d) Illustration of our hypothesis that the troughs align perpendicularly to expected polar wind trajectories, in order to explain the (counter-Coriolis) sense in which they are observed to spiral out from the pole. 


\subsection{Surface Evolution}

[10] Given today's polar conditions on Mars, we envisage a cold-based ice cap with its summer mass balance dominated by sublimation and by condensation directly onto the surface. If $\rho_{i}$ is ice density, and $\dot{m}$ is the mass sublimation rate normal to the surface ( $\dot{m}<0$ if water vapor condenses), then an idealized description of the cap evolution is

$$
\frac{\partial s}{\partial t}=\frac{1}{\eta_{i}} \frac{\partial^{2} s}{\partial x^{2}}-\frac{\dot{m}}{2 \rho_{i}} \sqrt{1+\left(\frac{\partial s}{\partial x}\right)^{2}} \quad(+M) .
$$

On the right-hand side of this equation, the first term mimics viscous ice flow (internal deformation) by parameterizing its diffusive effect on topography; $\eta_{i}$ is an effective ice viscosity. The second term represents mass balance due to $\dot{m}$ when it is cast in the vertical coordinate; the factor $1 / 2$ arises because $\dot{m}$ acts for roughly half a year (in the summer). Source $M$ (optional) accounts for any $\mathrm{H}_{2} \mathrm{O}$ incorporated and then left behind by the $\mathrm{CO}_{2}$ frost that seals the cap in winter. In this paper, equation (1) is preferred to a formal kinematic description, such as

$$
\frac{\partial s}{\partial t}=-u_{i} \frac{\partial s}{\partial x}+w_{i}-\frac{\dot{m}}{2 \rho_{i}} \sqrt{1+\left(\frac{\partial s}{\partial x}\right)^{2}} \quad(+M),
$$

in which the horizontal and vertical surface velocities, $u_{i}$ and $w_{i}$, have to be solved with ice flow thermomechanics [Paterson, 1994]. Such solution suffers substantial uncertainties in the bed position of the Mars ice caps [see Zuber et al., 1998] and their rheology [Durham et al., 2003]. Equation (1) is also adequate because we anticipate the instability to arise in $\dot{m}$. Unless sustained by sublimation, surface troughs must fill in by viscous flow (basal sliding is negligible for the cold-based ice). This picture is confirmed by a flow model of the north polar cap [Hvidberg, 2003], in which $\dot{m}>0$ at the troughs and $\dot{m}<0$ on smooth terrains are necessary to keep today's surface at steady state.

\subsection{Mass and Energy Transfer}

[11] Remote instruments have detected water column abundances of up to $\sim 100 \mathrm{pr} \mu \mathrm{m}$ (precipitable microns) in the summer north polar atmosphere [Farmer et al., 1976]. Suppose the $\mathrm{H}_{2} \mathrm{O}$ partial pressure over the model ice cap is $\phi(x, t)$ (Figure 2). Ice will sublime if $\phi$ is less than $p_{s}$, the saturation vapor pressure associated with the surface temperature $T$ locally, and we expect vapor to condense if $\phi>$ $p_{s}$. A recipe for the saturation pressure is the Clapeyron relation,

$$
p_{s}=c_{0} e^{-c_{1} / T}
$$

where $c_{0}$ and $c_{1}$ are constants (see the notation section). To calculate the sublimation rate $\dot{m}$ for evolving the surface, we adopt the model by Ivanov and Muhleman [2000] and write two equations for sublimation rate kinetics and surface energy balance: respectively,

$$
\dot{m}=D\left(p_{s}-\phi\right) \sqrt{\frac{m_{w}}{2 \pi k T}}
$$

$$
I_{0}(1-a) \cos \left[\theta(x)-\tan ^{-1} \frac{\partial s}{\partial x}\right]-\sigma T^{4}=\dot{m} L
$$

where the variable $a$ denotes the surface albedo, $D$ is a rate factor (poorly constrained to within an order of magnitude at best), and other symbols are the $\mathrm{H}_{2} \mathrm{O}$ molecular mass $m_{w}$, Boltzmann constant $k$, incident solar flux $I_{0}$, StefanBoltzmann constant $\sigma$, and latent heat of vaporization $L$. Equation (3), whose form $\dot{m} \propto p_{s}-\phi$ relates the mass flux directions expected for undersaturation and oversaturation, has been derived from kinetic theory and shown to be consistent with earlier parameterizations [see Ivanov and Muhleman, 2000]. Equation (4) equates received radiation (first term) to reradiation (second term) and the heat involved in phase change (third term); it accounts for slope effects by including $\tan ^{-1} \partial s / \partial x$ as part of the incidence angle.

[12] Equations (2), (3), and (4) characterize the feedbacks that may destabilize the mass balance spatially, the crux to forming troughs. They describe how the surface in a known atmosphere responds (instantaneously) in terms of sublimation rate, temperature, and the associated saturation pressure; that is, they define $\dot{m}, T$, and $p_{s}$ implicitly as functions of the albedo $a$, vapor pressure $\phi$, and slope $\partial s / \partial x$. The most important function to us is $\dot{m}(a, \phi, \partial s / \partial x)$. Of its three arguments, each may destabilize $\dot{m}$ if $\dot{m}$ feedbacks on the argument's evolution, and a slope feedback via equation (1) may already be appreciated. To close the model we consider how $a$ and $\phi$ evolve.

\subsection{Albedo Regulation by Surface Dust}

[13] That the smooth terrains are bright and troughs are dark (Figure 1) suggests a feedback whereby surface albedo $a$ becomes bistable (and coupled to topography). Kieffer et al. [1976] attributed this to "cold-trapping", where the low temperature of a bright surface allows it to stay bright by causing ice to deposit on it. We suggest also that dark surfaces may remain dark, or darken, if their high temperature causes sublimation, exhuming dust to the surface from within the ice, whose dirtiness is betrayed by dark bands at the scarps [Laskar et al., 2002; Milkovich and Head, 2005].

[14] To model both processes, consider $a$ as depending on the area-fraction of dust resting on the surface, $f$, ranging from 0 to 1 . The albedo may be expressed as a weighted sum,

$$
a(f)=f a_{\mathrm{D}}+(1-f) a_{\mathrm{I}},
$$

where $a_{\mathrm{D}}$ is the dust albedo and $a_{\mathrm{I}}$ is the albedo of the cap ice; thus $f=0$ and $f=1$ describe the bright state and the dark state respectively. This model distinguishes between surface dust, represented by $f$, and dust in the ice, which influences $a_{\mathrm{I}}$. We take $a_{\mathrm{D}}=0.25$ and $a_{\mathrm{I}}=0.4$, values typical of the darkest and brightest areas in the north [Kieffer et al., 1976; Kieffer, 1990]. (The low value of $a_{\mathrm{I}}$ compared to terrestrial snow albedos (up to $\sim 0.9$ [Paterson, 1994]) may reflect the dirtiness of Martian ice, even if freshly deposited, although $a_{\mathrm{I}}$ depends also on radiative scattering by ice grains [Kieffer, 1990].) As pictured above, the surface brightens as it becomes buried by ice when $\dot{m}<0$ and darkens as dust accumulates on it when $\dot{m}>0$. If, in either case, $\Delta$ is the ice 
thickness change needed to bring $f$ from one end of its range to the other, then an approximate evolution equation for the dust fraction is $\partial f / \partial t=\dot{m} / \rho_{i} \Delta$. We propose a similar equation,

$$
\frac{\partial f}{\partial t}=\frac{\dot{m}}{\rho_{i} \Delta}-g_{0}\left(f-f_{0}\right), \quad \text { with } 0 \leq f \leq 1,
$$

which has a "dust stabilization" term, explained below. $\Delta$ generally differs for $\dot{m}<0$ and $\dot{m}>0$, and can be derived (with further assumptions) in these cases by modeling radiation attenuation in ice and by dividing the dust grain size by its volume concentration in ice, respectively. For convenience, however, we adopt a single $\Delta$ based on timescale consideration (section 5).

[15] We envisage continual exchange of dust between air and ground on Mars. A clear windy atmosphere may strip dust from dark surfaces $(\partial f / \partial t<0$ if $f$ is large); at the same time, topographic lows on the small-scale roughness of the surface will trap atmospheric dust, so that $f$ increases on the brightest, dust-free areas $(\partial f / \partial t>0$ if $f=0)$. The last term of equation (6) models the long-term effect of these exchanges by postulating that, independent of the effect of $\dot{m}$ discussed above, $f$ stabilizes exponentially to $f_{0}$ at the timescale $1 / g_{0}$. (The stripping and trapping rates balance when $f=f_{0}$.) Although the parameters $f_{0}$ and $g_{0}$ (both $>0$ ) depend in complicated ways on factors like wind speed, they can be adjusted to explore the effect of different dust-exchange regimes. If $f_{0}>1$, surfaces that may only receive atmospheric dust are assumed; that is, they are inflational. However, as we shall see, the interesting case is $0<f_{0}<$ 1 , as instability requires surfaces to be able to deflate (when $f>f_{0}$ ) as well as inflate (when $f<f_{0}$ ).

[16] Subsequently, we describe evolving albedos in terms of $f$, bearing in mind its direct conversion to $a$ by equation (5). The albedo at the equilibrium dust fraction $f_{0}$ is

$$
a_{0}=a\left(f_{0}\right)=f_{0} a_{\mathrm{D}}+\left(1-f_{0}\right) a_{\mathrm{I}} .
$$

\subsection{Moisture in the Atmosphere}

[17] Within a certain height above the surface, the pressure $\phi(x, t)$ responds markedly as sublimation (condensation) enriches (depletes) the air in moisture. We idealize this layer as vertically well mixed, having a constant height $h$, constant temperature $T_{0}$, and moving at a given wind velocity $u$ (Figure 2 ).

[18] If we ignore exchange with the upper atmosphere and write density using the ideal gas law, moisture conservation in the layer requires

$$
\left(\frac{\partial}{\partial t}+u \frac{\partial}{\partial x}-D_{v} \frac{\partial^{2}}{\partial x^{2}}\right)\left(\frac{h m_{w}}{k T_{0}} \phi\right)=\dot{m}
$$

where $D_{v}$ is the diffusion coefficient of $\mathrm{H}_{2} \mathrm{O}$ in the Mars atmosphere (which is mostly $\mathrm{CO}_{2}$ at a surface pressure of $\sim 6$ mbar). This model accounts for moisture transport and diffusion, but introduces $h / T_{0}$, a boundary-layer parameter which we will not further derive here. Nevertheless, a simple check below with equation (8) shows that our instability can create patterns at the right scale; we set $T_{0}$ to a typical temperature of the problem (equation (15)), then adjust $h$ to match the predicted trough spacing to the observed one, with the proviso that $h$ lies between the surface roughness (meters or less) and the troposphere height ( $\sim 50 \mathrm{~km})$. Equations (1) to (8) complete the model.

\section{Oscillations and Pattern Formation}

[19] Winds are important not only in our hypothesis of spiraling. They also control the proposed instability whose characteristics, as we shall see, depend on wind transport. The mechanism hinges on two competing feedbacks. A positive albedo feedback (on $a$ and $f$ ) has been mentioned before. When $\dot{m}<0$, brightening of the surface depresses its temperature, lowering the atmosphere's capacity to hold moisture (the saturation pressure $p_{s}$ ), and thereby increasing the oversaturation $\phi-p_{s}$ and the condensation rate. When $\dot{m}>0$, the surface darkens and warms, and an increased $p_{s}$ (and $p_{s}-\phi$ ) accelerates sublimation. These runaways could in principle lead to $f=0$ or $f=1$. However, they are opposed by a second feedback as $\phi$ varies: Whether $\dot{m}<0$ or $\dot{m}>0$, vapor depletion or enrichment will cause the atmosphere to evolve toward saturation, with $\dot{m}$ diminishing as $\phi$ approaches $p_{s}$; this vapor feedback is negative.

[20] The coupling of dust fraction and vapor pressure is easy to study in a temporal version of equations (6) and (8) where we neglect winds and gradients $(u=\partial / \partial x=0)$. We have

$$
\begin{gathered}
\frac{\mathrm{d} f}{\mathrm{~d} t}=\frac{1}{\rho_{i} \Delta} \dot{m}(f, \phi, 0)-g_{0}\left(f-f_{0}\right), \quad \text { with } 0 \leq f \leq 1, \\
\frac{\mathrm{d} \phi}{\mathrm{d} t}=\frac{k T_{0}}{h m_{w}} \dot{m}(f, \phi, 0),
\end{gathered}
$$

where $\dot{m}(f, \phi, 0)$ is the zero-slope sublimation rate (Figure 3a). This nonlinear system has an activator $f$ and an inhibitor $\phi$ due to the signs of the feedbacks on these variables [Murray, 2002, section 7.3], governed by $\partial \dot{m} / \partial f>$ 0 and $\partial \dot{m} / \partial \phi<0$ (general properties of $\dot{m}$ ). Figures $3 \mathrm{~b}$ and $3 \mathrm{c}$ show the system's phase portrait assuming $g_{0}$ to be small for the albedo feedback to overcome dust stabilization, and assuming $0<f_{0}<1$, so that the system has its steady state $\mathrm{P}$ in $0<f<1$. ( $\mathrm{P}$ is the intersection point of two curves, called nullclines (dashed lines), defined, respectively, by $\mathrm{d} f / \mathrm{d} t=0$ and $\mathrm{d} \phi / \mathrm{d} t=0$.) We find two types of behavior depending on which feedback is faster. If the albedo feedback dominates (Figure $3 \mathrm{~b}$ ), $f$ and $\phi$ oscillate unstably in a spiral trajectory that turns into a limit cycle when $f$ reaches its limits. If the vapor feedback dominates (Figure 3c), $\phi$ reaches saturation (the $\phi$-nullcline) quickly and the system settles at P. In terms of an albedo (evolution) timescale $t_{\mathrm{A}} \sim \rho_{i} \Delta / \dot{m}$ and a vapor (evolution) timescale $t_{\mathrm{V}} \sim h m_{w} \phi / k T_{0} \dot{m}$, these considerations imply instability if $\epsilon=t_{\mathrm{V}} / t_{\mathrm{A}}$ exceeds a parameter $\epsilon_{\mathrm{c}}(\sim 1)$. Later, analysis shows that $\epsilon_{\mathrm{c}}$ depends on the form of $\dot{m}$ and that the precise instability condition is

$$
\frac{h m_{w}}{k T_{0} \rho_{i} \Delta}>\frac{(-\partial \dot{m} / \partial \phi)_{\mathrm{P}}}{(\partial \dot{m} / \partial f)_{\mathrm{P}}-g_{0} \rho_{i} \Delta},
$$



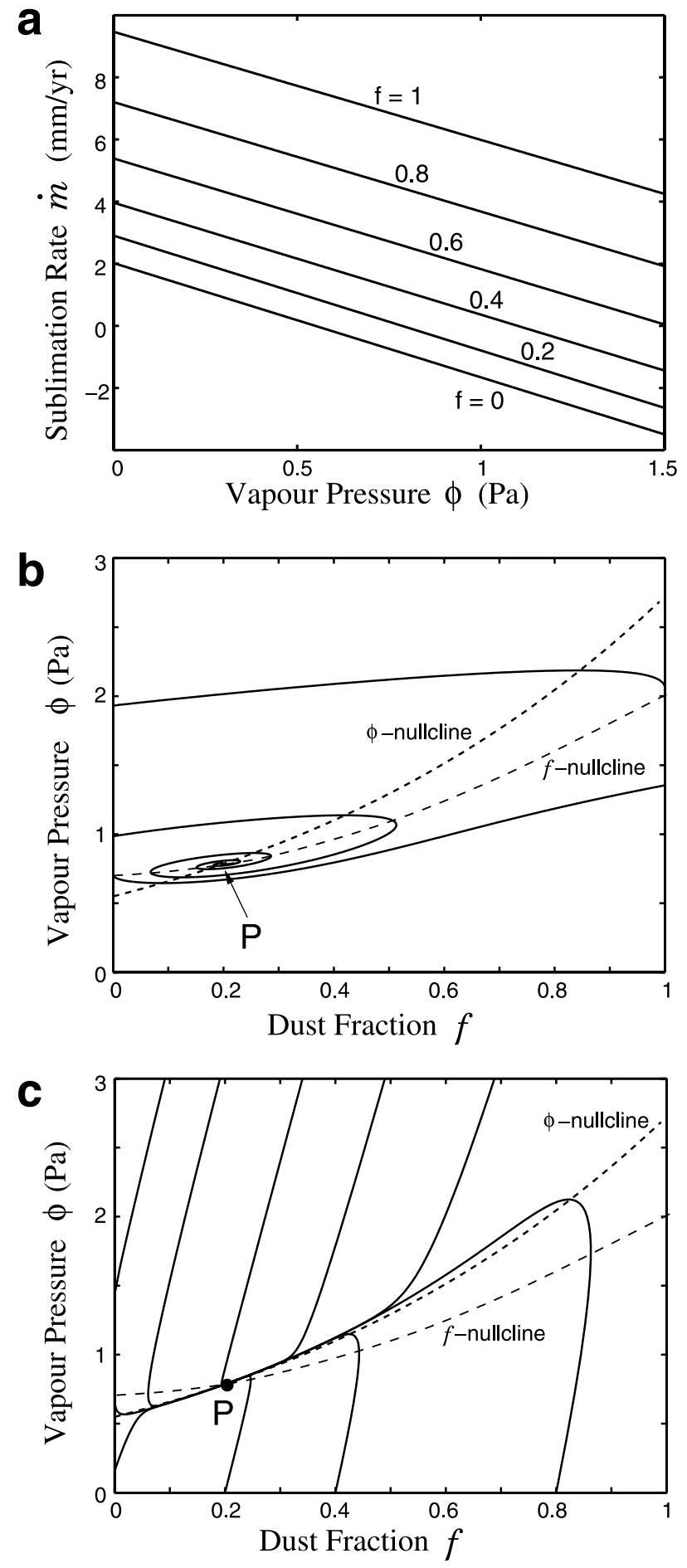

Figure 3. (a) Sublimation rate $\dot{m}$ as a function of vapor pressure $\phi$ on lines of constant dust fraction $f$ (which controls surface albedo) for a horizontal ice surface. (b) Phase portrait $(f, \phi)$ of equations (9) and (10) when the albedo feedback dominates $(\epsilon=2)$. It shows an unstable trajectory spiraling out from the point P. (c) Phase portrait $(f, \phi)$ when the vapor feedback dominates $(\epsilon=0.05)$. It shows stable trajectories that approach $\mathrm{P}$ after meeting the $\phi$-nullcline. Both phase portraits are calculated from the dimensionless model assuming $f_{0}=0.2, \gamma=1$, and a zenith angle $72.5^{\circ}$. together with

$$
g_{0} \rho_{i} \Delta<(\partial \dot{m} / \partial f)_{\mathrm{P}}
$$

(the definition of $g_{0}$ being small). Note that model oscillations involve cycles of air drying and wetting and cycles of dust accumulation and removal. None will occur if the surface is inflational; if $f_{0}>1$, the phase trajectories cannot revolve around $\mathrm{P}$.

[21] The coupling is fundamentally changed by winds in the spatial extension of equations (9) and (10). On Mars, $\epsilon$ is in fact very small, $\lesssim 10^{-4}$ (Table 1 ); $\phi$ evolves much faster than $f$ because the atmosphere contains $10^{2} \mathrm{pr} \mu \mathrm{m} \mathrm{H}_{2} \mathrm{O}$ at most, and sublimation or condensation that has little effect on $f$ can drive large changes in $\phi$. The purely temporal system is stable. (Indeed, we do not see episodic switching of polar cap albedo.) However, instability may occur if wind carries moisture over the surface. In this case, $\phi$ depends not so much on the vapor feedback as on the history of an air packet over surfaces upwind, whose drying-wetting rate evolves on the albedo timescale. Disengaging the local coupling of $\phi$ and $f$ puts $\phi$ on this timescale, effectively making $\epsilon$ much larger and allowing a moderate wind velocity to activate instability; this is our proposed mechanism. As a pattern subsequently evolves, $f$ and $\phi$ interact in a way that is difficult to visualize. We return to the full model and analyze its spatial dynamics next.

\section{Dimensionless Model}

[22] Because the model is burdened by many physical constants, we nondimensionalize equations (1) to (8) by using the scales $[t],[x],[s],[\dot{m}],\left[p_{s}\right],[T],[\phi]$, and

Table 1. Scales and Parameters of our Dimensionless Model ${ }^{\mathrm{a}}$

\begin{tabular}{lll}
\hline \multicolumn{1}{c}{ Symbol } & \multicolumn{1}{c}{ Value } & \multicolumn{1}{c}{ Unit } \\
\hline & Scales & \\
{$[T], T_{0}$} & 210.7 & $\mathrm{~K}$ \\
{$[\phi],\left[p_{s}\right]$} & 0.79 & $\mathrm{~Pa}^{\mathrm{b}}$ \\
{$[\dot{m}]$} & $8.3 \times 10^{-8}$ & $\mathrm{~kg} \mathrm{~m}^{-2} \mathrm{~s}^{-1}$ \\
{$[s]$} & 10 & $\mathrm{~km}^{-1}$ \\
& & \\
$h$ & Parameters & $\mathrm{m}$ \\
$c$ & 103 & \\
$\beta$ & 29.1 & \\
$\xi$ & $2.1 \times 10^{-3}$ & $\mathrm{~m}$ \\
$\mathrm{Pe}$ & 0.238 & $\mathrm{~m}$ \\
$\Delta,[t]=10^{0}$ years & $10-7$ & \\
$\Delta,[t]=10^{3}$ years & $2.9 \times 10^{-3}$ & \\
$\delta,[t]=10^{0}$ years & 2.9 & \\
$\delta,[t]=10^{3}$ years & $3 \times 10^{-7}$ & \\
$\epsilon,[t]=10^{0}$ years & $3 \times 10^{-4}$ & \\
$\epsilon,[t]=10^{3}$ years & $3 \times 10^{-4}$ & \\
\hline
\end{tabular}

${ }^{\mathrm{a}}$ On the basis of $f_{0}=0.2$ (whereby equation (7) gives $a_{0}=0.37$ ), $D=$ $8.25 \times 10^{-5}$ (the upper end of the range by Ivanov and Muhleman [2000]), and two conservative end values of the albedo timescale $[t]$. The (estimated) moisture layer thickness $h$ falls in the range between the surface roughness and the troposphere height. Lowering $D$ reduces $[\dot{m}], h, \beta, \Delta$, and $\delta$ proportionately.

${ }^{\mathrm{b}} \mathrm{A}$ surface vapor partial pressure of $0.1 \mathrm{~Pa}$ corresponds roughly to $\mathrm{O}(10)$ pr $\mu \mathrm{m} \mathrm{H}_{2} \mathrm{O}$ in terms of column abundance [e.g., Jakosky and Phillips, 2001]. The exact conversion depends on the vertical distribution of atmospheric water vapor. 
$[u]$, defined for those variables inside brackets, writing $t=$ $t^{*}[t], x=x^{*}[x]$, and so on, where the asterisks label scaled variables. (The variables $f$ and $a$ are dimensionless.) We also choose a reference zenith angle for the polar summer (the precise value is not critical),

$$
\theta_{0}=72.5^{\circ}
$$

then, using the observed trough spacing and a wind speed that may be typical of Mars, we let

$$
[x]=10 \mathrm{~km}, \quad[u]=1 \mathrm{~m} \mathrm{~s}^{-1},
$$

and apply the formulae

$$
\begin{aligned}
& I_{0}\left(1-a_{0}\right) \cos \theta_{0}=\sigma[T]^{4}, \quad T_{0}=[T], \quad[\phi]=\left[p_{s}\right]=c_{0} e^{-c_{1} /[T]}, \\
& {[\dot{m}]=D[\phi] \sqrt{\frac{m_{w}}{2 \pi k[T]}}, \quad[x]=[s]=\frac{h m_{w}[u][\phi]}{k[T][\dot{m}]}, \quad[t]=\frac{\rho_{i} \Delta}{[\dot{m}]}}
\end{aligned}
$$

to find the other scales, $T_{0}$, and an estimate for $h$. We prescribe the albedo timescale $[t]$ to back out $\Delta$ instead of the other way round. The northern cap albedo exhibits seasonal and annual variability [Bass et al., 2000; James and Cantor, 2001; Calvin and Titus, 2004; Langevin et al., $2005]$ suggesting $[t] \sim 10^{0}$ year to be plausible. Here we suppose the range

$$
[t]=10^{0} \text { to } 10^{3} \text { year, }
$$

whose high end value will demonstrate that various approximations used later are robust.

[23] When converted to dimensionless form, the evolution equations (1), (6), and (8) become

$$
\begin{aligned}
& \delta^{-1} \frac{\partial s}{\partial t}=\kappa \frac{\partial^{2} s}{\partial x^{2}}-\frac{\dot{m}}{2} \sqrt{1+\left(\frac{\partial s}{\partial x}\right)^{2}} \quad(+M), \\
& \frac{\partial f}{\partial t}=\dot{m}-\gamma\left(f-f_{0}\right), \quad \text { with } 0 \leq f \leq 1, \\
& \epsilon \frac{\partial \phi}{\partial t}+u \frac{\partial \phi}{\partial x}=\dot{m}+\mathrm{Pe}^{-1} \frac{\partial^{2} \phi}{\partial x^{2}}
\end{aligned}
$$

(we drop asterisks with the understanding that all variables have now been scaled), and equations (2), (3), and (4) (for the sublimation rate) become

$$
\begin{aligned}
& p_{s}=e^{c\left(1-\frac{1}{T}\right)}, \\
& \dot{m}=\frac{p_{s}-\phi}{\sqrt{T}}, \\
& G_{1}(f) G_{2}(x, s)-T^{4}=\beta \dot{m},
\end{aligned}
$$

where $G_{1}$ (representing radiation absorption) and $G_{2}$ (representing latitude and slope effects) are order-one functions given by

$$
\begin{gathered}
G_{1}(f)=\frac{1-a(f)}{1-a_{0}}=1+\frac{a_{0}-a(f)}{1-a_{0}}=1+\xi\left(f-f_{0}\right), \\
G_{2}(x, s)=\frac{\cos \left(\theta(x)-\tan ^{-1} \partial s / \partial x\right)}{\cos \theta_{0}} .
\end{gathered}
$$

The dimensionless parameters in this model are

$$
\begin{aligned}
& \delta=\frac{\Delta}{[x]}, \quad \kappa=\frac{[t]}{\eta_{i} \Delta[x]}, \quad \gamma=g_{0}[t], \quad \epsilon=\frac{[x]}{[u][t]}, \\
& \mathrm{Pe}^{-1}=\frac{D_{v}}{[u][x]}, \quad c=\frac{c_{1}}{[T]}, \quad \beta=\frac{[\dot{m}] L}{\sigma[T]^{4}}, \quad \xi=\frac{a_{\mathrm{I}}-a_{\mathrm{D}}}{1-a_{0}} .
\end{aligned}
$$

Table 1 gives scales and parameters calculated with the values in the notation section and with an equilibrium dust fraction $f_{0}=0.2$, omitting $\kappa$ (which represents ice flow diffusivity) and $\gamma$ (which represents dust stabilization rate) as these will be kept variable.

[24] Besides being attached to sensible scales of the problem, the dimensionless model (equations (17) to (24)) represents the original model efficiently. Table 1 identifies small parameters that are useful for simplifying it. For instance, $\beta \ll 1$ means that latent heat is negligible in the energy balance; we can write equations (20) to (22) as $\dot{m}=$ $T^{-1 / 2}\left(\exp \left[c\left(1-T^{-1}\right)\right]-\phi\right)$, with $T \approx\left(G_{1} G_{2}\right)^{1 / 4}$ (which is independent of $\phi)$. Thus the sublimation rate should decrease linearly with $\phi$, and we see this in Figure $3 \mathrm{a}$.

[25] More important are the parameters $\epsilon$ and $\delta$. Like $\epsilon \ll 1$, whose meaning we discussed before, $\delta \ll 1$ distinguishes different timescales of the model; it indicates the exceedingly slow evolution of surface topography as $f$ and $\phi$ vary. Because of this, the wind-activated instability described in section 4 will form patterns much faster than a slopedriven instability (if viable) can initiate troughs. Table 1 shows that the ordering of topography, albedo, and vapor timescales $\left(\delta^{-1} \gg 1 \gg \epsilon\right)$ holds over a large range of the assumed albedo timescale $[t]$. This means that the process of trough development may be broken into two stages: "fast" albedo patterning (section 6.2) and "slow" surface evolution (section 6.3).

\section{Patterning Dynamics}

\subsection{Linear Stability}

[26] First, we need to show that the model can indeed become unstable and to derive general instability conditions. To do this, we perturb equations (17) to (24) about their steady state $s=\dot{m}=0, f=f_{0}(<1), \phi=p_{s}=T=1$ by writing

$$
\begin{aligned}
s & =0+\tilde{s}, \quad \dot{m}=0+\tilde{m}, \quad f=f_{0}+\tilde{f}, \quad \phi=1+\tilde{\phi}, \\
p_{s} & =1+\tilde{p_{s}}, \quad T=1+\tilde{T}
\end{aligned}
$$


(squiggles label small perturbations), linearizing them to find

$$
\begin{aligned}
& \delta^{-1} \tilde{s}_{t}=\kappa \tilde{s}_{x x}-0.5 \tilde{m}, \\
& \tilde{f}_{t}=\tilde{m}-\gamma \tilde{f}, \\
& \epsilon \tilde{\phi}_{t}+u \tilde{\phi}_{x}=\tilde{m}+\operatorname{Pe}^{-1} \tilde{\phi}_{x x}, \\
& \tilde{p_{s}}=c \tilde{T}, \\
& \tilde{m}=\tilde{p_{s}}-\tilde{\phi}, \\
& \xi \tilde{f}+\left(\tan \theta_{0}\right) \tilde{s}_{x}-4 \tilde{T}=\beta \tilde{m} .
\end{aligned}
$$

Eliminating $\tilde{T}$ and $\tilde{p}_{s}$ from the last three equations gives

$$
\tilde{m}=\nu_{1} \tilde{f}+\nu_{2} \tilde{\phi}+\nu_{3} \tilde{s}_{x}
$$

in which the gradients (evaluated about the steady state)

$\nu_{1}=\frac{\partial \dot{m}}{\partial f}=\frac{c \xi}{4+\beta c}, \quad \nu_{2}=\frac{\partial \dot{m}}{\partial \phi}=\frac{-4}{4+\beta c}$,

$\nu_{3}=\frac{\partial \dot{m}}{\partial s_{x}}=\frac{c \tan \theta_{0}}{4+\beta c}$

describe how the sublimation rate responds to changes in dust fraction, pressure, and slope $\left(\nu_{1}>0, \nu_{2}<0, \nu_{3}>0\right.$, and Table 1 yields $\left.\nu_{1}=1.705, \nu_{2}=-0.985, \nu_{3}=22.726\right)$. If we expand the perturbations as Fourier components of the form $\exp (\lambda t+i k x)$, where the wavenumber $k$ is real, then the first three equations in (26) together with equation (27) yield the characteristic equation

$$
\left|\begin{array}{ccc}
\lambda-\left(\nu_{1}-\gamma\right) & -\nu_{2} & -i k \nu_{3} \\
-\nu_{1} & \epsilon \lambda-\nu_{2}+i k u+\mathrm{Pe}^{-1} k^{2} & -i k \nu_{3} \\
0.5 \nu_{1} & 0.5 \nu_{2} & \delta^{-1} \lambda+0.5 i k \nu_{3}+\kappa k^{2}
\end{array}\right|=0
$$

for the eigenvalue $\lambda$. In the purely temporal case $k=0$, this equation becomes

$$
\lambda\left[\epsilon \lambda^{2}-\lambda\left[\nu_{2}+\epsilon\left(\nu_{1}-\gamma\right)\right]-\gamma \nu_{2}\right]=0,
$$

and the growth rate of perturbations $\operatorname{Re} \lambda$ can be positive (indicating instability) only if $\nu_{2}+\epsilon\left(\nu_{1}-\gamma\right)>0$, or equivalently,

$$
\nu_{1}-\gamma>0 \quad \text { and } \quad \epsilon>\epsilon_{\mathrm{c}}=\frac{-\nu_{2}}{\nu_{1}-\gamma} .
$$

In dimensioned terms, these are the conditions in equations (12) and (11).

[27] To determine how $\operatorname{Re} \lambda$ varies with the wavenumber $k$, we have solved equation (29) approximately with asymptotics based on $\delta, \epsilon \ll 1$. Omitting the algebra, we simply state that the leading order results for $\lambda=\lambda(k)$ (the dispersion relations) are

$$
\begin{aligned}
\lambda_{1}= & -\delta k^{2}\left[\kappa+\left(\frac{\gamma \nu_{3}}{2}\right) \frac{u \gamma \nu_{2}-i k\left[\left(\nu_{1}-\gamma\right)\left(u^{2}+\mathrm{Pe}^{-2} k^{2}\right)+\mathrm{Pe}^{-1} \gamma \nu_{2}\right]}{\left[\gamma \nu_{2}+\mathrm{Pe}^{-1}\left(\nu_{1}-\gamma\right) k^{2}\right]^{2}+\left(\nu_{1}-\gamma\right)^{2} u^{2} k^{2}}\right] \\
& +\mathrm{O}\left(\delta^{2}, \epsilon \delta\right) \text { terms }
\end{aligned}
$$
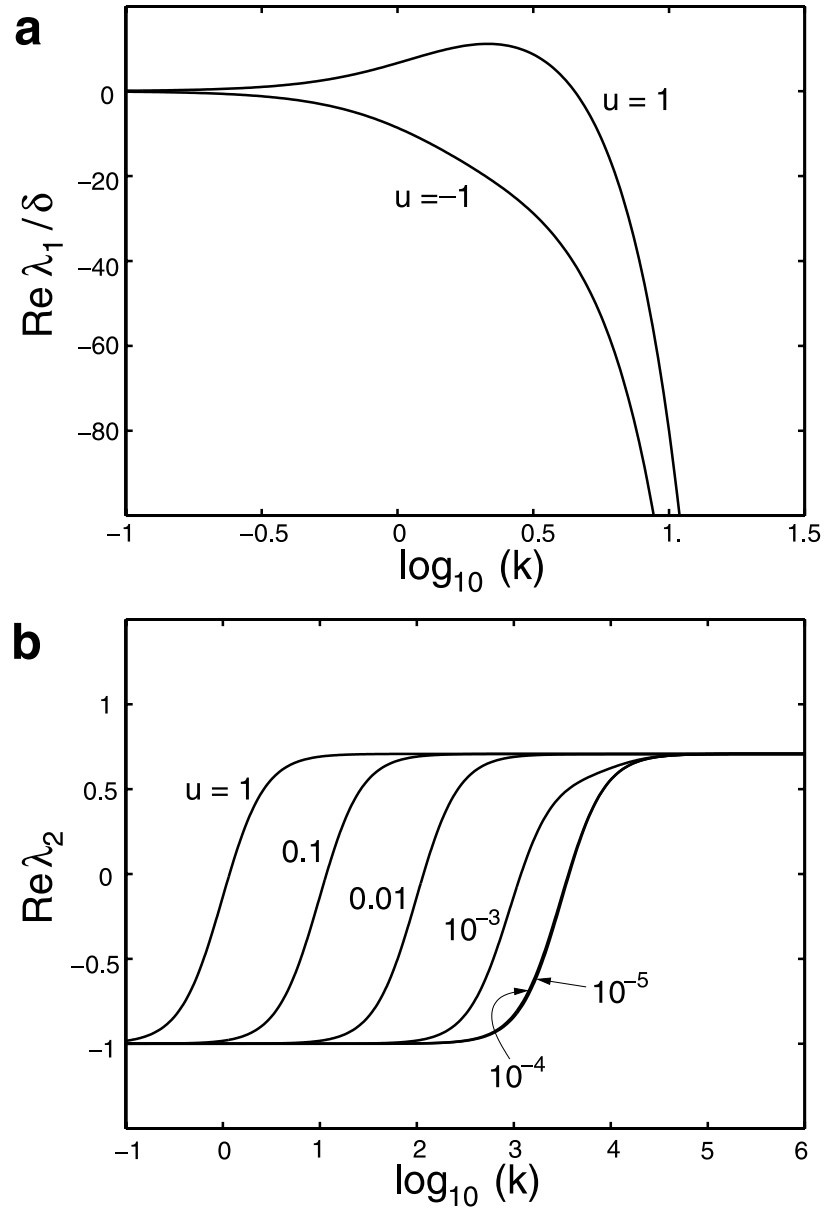

Figure 4. Dispersion relations from our linear stability analysis with $\gamma=1, \kappa=1$, and values of $\nu_{1}, \nu_{2}, \nu_{3}$, and Pe derived from Table 1 . (a) $\operatorname{Re} \lambda_{1}(k) / \delta$ for $u=1$ (poleward wind) and for $u=-1$ (equatorward wind). (b) $\operatorname{Re} \lambda_{2}(k)$ for six different wind speeds indicate a Turing instability at high $k$ and a wind-activated transport instability at low $k$, regardless of wind direction. The lowest unstable wavenumber $k_{c}(u)$ may be found by setting $\operatorname{Re} \lambda_{2}(k)=0$ in equation (33) and solving for $k$.

$$
\begin{aligned}
\lambda_{2}= & \frac{\left(\nu_{1}-\gamma\right) u^{2} k^{2}+\left[\mathrm{Pe}^{-1} k^{2}-\nu_{2}\right]\left[\gamma \nu_{2}+\mathrm{Pe}^{-1}\left(\nu_{1}-\gamma\right) k^{2}\right]-i k u \nu_{1} \nu_{2}}{\left(\nu_{2}-\mathrm{Pe}^{-1} k^{2}\right)^{2}+k^{2} u^{2}} \\
& +\mathrm{O}(\delta, \epsilon) \text { terms, }
\end{aligned}
$$

$$
\lambda_{3}=\frac{\left(\nu_{2}-\mathrm{Pe}^{-1} k^{2}\right)-i k u}{\epsilon}+\mathrm{O}(\delta / \epsilon, 1) \text { terms }
$$

Here $\nu_{2}<0$ implies $\operatorname{Re} \lambda_{3}(k)<0$ and no unstable mode in the (fast) vapor timescale. On the other hand, three types of instability are predicted by the expressions for $\lambda_{1}$ and $\lambda_{2}$.

\subsubsection{Type I}

[28] While $\operatorname{Re} \lambda_{1} \rightarrow-\delta \kappa k^{2}<0$ as $k \rightarrow \infty$, we have, for $k \approx 0$, Re $\lambda_{1} \approx-\delta k^{2}\left(\kappa+u \nu_{3} / 2 \nu_{2}\right)$, which is positive if $u \nu_{3}>-2 \kappa \nu_{2}$. A long-wave instability thus operates on 

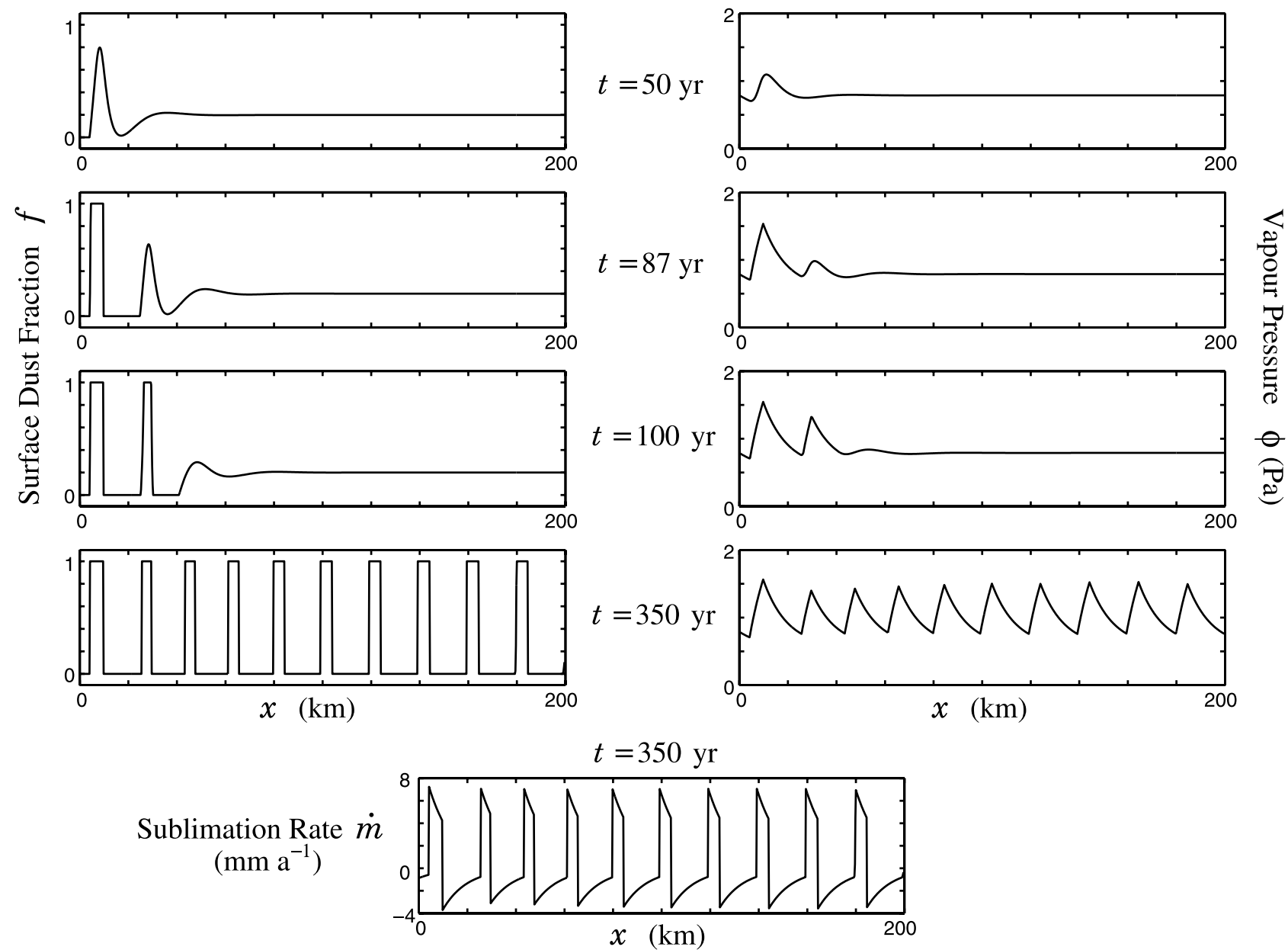

Figure 5. (top) Propagation of an oscillatory front in (left) dust fraction and (right) vapor pressure over polar ice at four times in the simulation described in section 6.2, which assumes model parameters $u=\gamma=1$ and $f_{0}=0.2$. The front leaves behind an alternating albedo pattern $(f=0$ indicates high albedo, $f=1$ indicates low albedo) associated with which is a saw-tooth pattern in vapor pressure. The final pattern is stable owing to positive albedo feedback on the surface mass balance. (bottom) Sublimation rate at $t=$ 350 years expressed as ice thickness sublimed per year. For comparison, equation (42) predicts the (theoretical) pattern spacing to be $\Lambda=2.05$ or, dimensionally, $20.5 \mathrm{~km}$.

the slow topography timescale via slope feedback, if a poleward wind blows (e.g., Figure 4a).

[29] Much faster and more important than Type I are two kinds of instabilities on the albedo timescale: Type IIa and Type IIb.

\subsubsection{Type IIa}

[30] The first of these is our wind-activated transport instability (section 4). According to equation (33), it dominates at wavelengths comparable to the observed trough spacing when $|u| \sim 1$; then terms in $\mathrm{Pe}^{-1}$ are negligible, and the condition $\operatorname{Re} \lambda_{2}>0$ is met by $\left(\nu_{1}-\gamma\right) u^{2} k^{2} \gtrsim \gamma \nu_{2}^{2}$, or $\gamma<\nu_{1}$ (the dust stabilization being weak) and

$$
k>k_{\mathrm{c}} \approx \frac{-\nu_{2}}{|u|} \sqrt{\frac{\gamma}{\nu_{1}-\gamma}} .
$$

The above approximation for the lowest unstable wavenumber, $k_{\mathrm{c}}$, needs to be revised if $|u|$ is small, due to the operation of a second, fast instability (next).

\subsubsection{Type IIb}

[31] If $|u| \approx 0$, then $\operatorname{Re} \lambda_{2}>0$ if $\left[\gamma \nu_{2}+\operatorname{Pe}^{-1}\left(\nu_{1}-\gamma\right) k^{2}\right] \gtrsim 0$ in equation (33), predicting instability at high wavenumbers,

$$
k>k_{\mathrm{c}} \approx \sqrt{\frac{-\gamma \nu_{2}}{\mathrm{Pe}^{-1}\left(\nu_{1}-\gamma\right)}}
$$

if again $\gamma<v_{1}$. Studied by Turing [1952] for chemical pattern formation, this instability occurs because diffusion (of vapor) disengages the inhibitor $(\phi)$ from the activator $(f)$ to allow the latter to grow. The Turing instability is a permanent feature of our model at short length scales below $2 \pi / k_{c}(\approx 20 \mathrm{~m}$ if, say, $\gamma=1$ and we use the values of Table 1); whereas, if a wind blows, the transport mechanism (Type IIa) enables instability at longer wavelengths. Figure $4 \mathrm{~b}$ shows this behavior by plotting $\operatorname{Re} \lambda_{2}(k)$ for various wind velocities. 
[32] If the steady state is unstable, patterns must form. Before we examine them, however, comments are in order for the positive growth rate at the short-wave limit (see Figure 4b). Not only does $\operatorname{Re} \lambda_{2}$ indicate no preferred instability wavelength (no maximum at finite $k$ ), $\operatorname{Re} \lambda_{2} \rightarrow$ $v_{1}-\gamma$ as $k \rightarrow \infty$ means that patterns emerging at the trough length scale could be swamped by fast-growing short perturbations. Because the transport and Turing mechanisms share the same limiting growth rate, this problem is not necessarily solved by reparameterizing the 1-D vapor diffusion in equation (19), which controls the latter mechanism only. The other option is to add artificial dust diffusion (e.g., a term $\chi \partial^{2} f / \partial x^{2}$ ) to equation (18) to stabilize high wavenumbers, but it requires faster diffusion of dust than of vapor $\left(\mathrm{x}>\mathrm{Pe}^{-1}\right)$ [Rovinsky and Menzinger, 1992] and is unrealistic. The solution here rests on the observations. For the type of models to which ours belongs, it is known that pattern develops either from noise, owing to the short-wave instability, or by the propagation of a wave front from the system boundary, owing to the transport instability [Walgraef, 1997, chapter 9]; in our case, it is the wave front which produces the observed albedo pattern (section 6.2). We naturally assume that even if the system on Mars were initially near the steady state, the noise on it was too small to grow significantly before the front passed by; otherwise we would see a very different, short-wave pattern today. Because this assumption precludes the Turing instability (Type IIb), from now we neglect the moisture diffusion term in equation (19) and set $\mathrm{Pe}^{-1}=0$.

\subsection{Albedo Pattern}

[33] Here we study the spatial dynamics of the model in the albedo timeframe by decoupling from it slow topography evolution, and by treating vapor equilibration as instantaneous; thus we take $\delta, \epsilon \rightarrow 0$ in equations (17) to (24). We assume windy conditions $|u| \sim 1$ and a flat surface without latitude effects $\left(G_{2}=1\right)$ for simplicity. On setting $\mathrm{Pe}^{-1}$ to zero, equations (18) and (19) become

$$
\begin{gathered}
f_{t}=\dot{m}(f, \phi)-\gamma\left(f-f_{0}\right), \quad 0 \leq f \leq 1, \\
u \phi_{x}=\dot{m}(f, \phi) .
\end{gathered}
$$

Integration of the last equation for $u>0$ (poleward wind) gives

$$
\phi(x, t)=\phi(x=0, t)+\frac{1}{u} \int_{0}^{x} \dot{m}[f(x, t), \phi(x, t)] d x
$$

which illustrates the idea that the vapor pressure $\phi$ depends on upwind sublimation history and varies on the same timescale as the dust fraction $f$. (The boundary condition is specified at the other end of the $x$-domain if $u<0$.) Our following experiment assumes $u=1$ and the parameters in Table 1 , adjusted for an albedo timescale $[t]=10$ years.

[34] In the toy model above, traveling waves arise because of the transport instability (Type IIa) [Rovinsky and Menzinger, 1992; Rovinsky et al., 1996]. Figure 5 shows a simulation of equations (37) and (38) with $f_{0}=0.2, \gamma=1$, a "bright" initial condition $f(x, 0)=0$, and a fixed vapor pressure $\phi=1$ at the ice margin $x=0$. (Anticipating discontinuities in $f$, we devised our finite difference scheme with $f$ centered on each grid element and $\phi$ at each grid point. The results are redimensioned using the scales of Table 1.) An oscillating front in $f$ and $\phi$ advances into the domain, leaving behind a stationary albedo pattern that alternates between bright and dark. The qualitative behavior of this solution is robust: If dust stabilization is weak $(\gamma<$ $\left.\nu_{1}\right)$, a pattern will form unless we set both the boundary and initial conditions deliberately to the steady state of the system.

[35] As Satnoianu and Menzinger [2000] described for a similar model for chemical systems, our patterning mechanism involves oscillations carried into space by (wind) transport and locked in phase at the boundary. However, the model here differs from the oscillator of section 4 for having a partial derivative, and hence its analysis is nontrivial. Nonlinearities due to the function $\dot{m}$ and cutoffs in $f$ also make predicting the final albedo pattern difficult. Notably, we cannot determine the width ratio of bright to dark regions without simulation, although it usually turns out to be large in our experiments, as is observed on the ice caps (Figure 1c).

[36] The pattern wavelength can be estimated. Figure 5 shows an irregular spacing in the first few cycles near $x=0$, but as this upwind boundary recedes far behind the moving front, the new pattern that forms becomes essentially periodic. The following method derives from an analysis of this regime, where the front speed becomes constant.

[37] As in other models where bistable states invade an unstable steady state to form pattern, our front is an "envelope front" [Walgraef, 1997]. At its leading edge we can write $f=f_{0}+\tilde{f}$ and $\phi=1+\tilde{\phi}$, where $\tilde{f}$ and $\tilde{\phi}$, the deviations from the steady state, can be taken as proportional to $\exp (\omega t-k x)$. Both the wavenumber $k$ and growth rate $\omega$ are complex, and we expect $\operatorname{Im} k \neq 0$ (the front oscillates in space) and $\operatorname{Re} k>0$ (the front envelope decays into the steady state). The front speed $c^{*}$ is given by Rew/ Rek.

[38] Now the linearized version of equations (37) and (38) are

$$
\begin{aligned}
\tilde{f}_{t} & =\left(\nu_{1}-\gamma\right) \tilde{f}+\nu_{2} \tilde{\phi}, \\
u \tilde{\phi}_{x} & =\nu_{1} \tilde{f}+\nu_{2} \tilde{\phi},
\end{aligned}
$$

and substituting the exponential solution into these yields the dispersion relation

$$
\omega(k)=\nu_{1}-\gamma-\frac{\nu_{1} \nu_{2}}{\nu_{2}+u k}
$$

A thorough discussion of how a pattern is selected based on $\omega(k)$ has been given by Dee and Langer [1983], van Saarloos [1987], Dee and van Saarloos [1988] and the related literature. The basic idea is that the selected front is such that slower fronts are convectively unstable and faster fronts are absolutely unstable, and consequently the front wavenumber $k_{0}$ and speed $c^{*}$ satisfy the equations

$$
\left.\operatorname{Im} \frac{\mathrm{d} \omega(k)}{\mathrm{d} k}\right|_{k_{0}}=0,\left.\quad \operatorname{Re} \frac{\mathrm{d} \omega(k)}{\mathrm{d} k}\right|_{k_{0}}=\frac{\operatorname{Re} \omega\left(k_{0}\right)}{\operatorname{Re} k_{0}}=c^{\star} .
$$


a

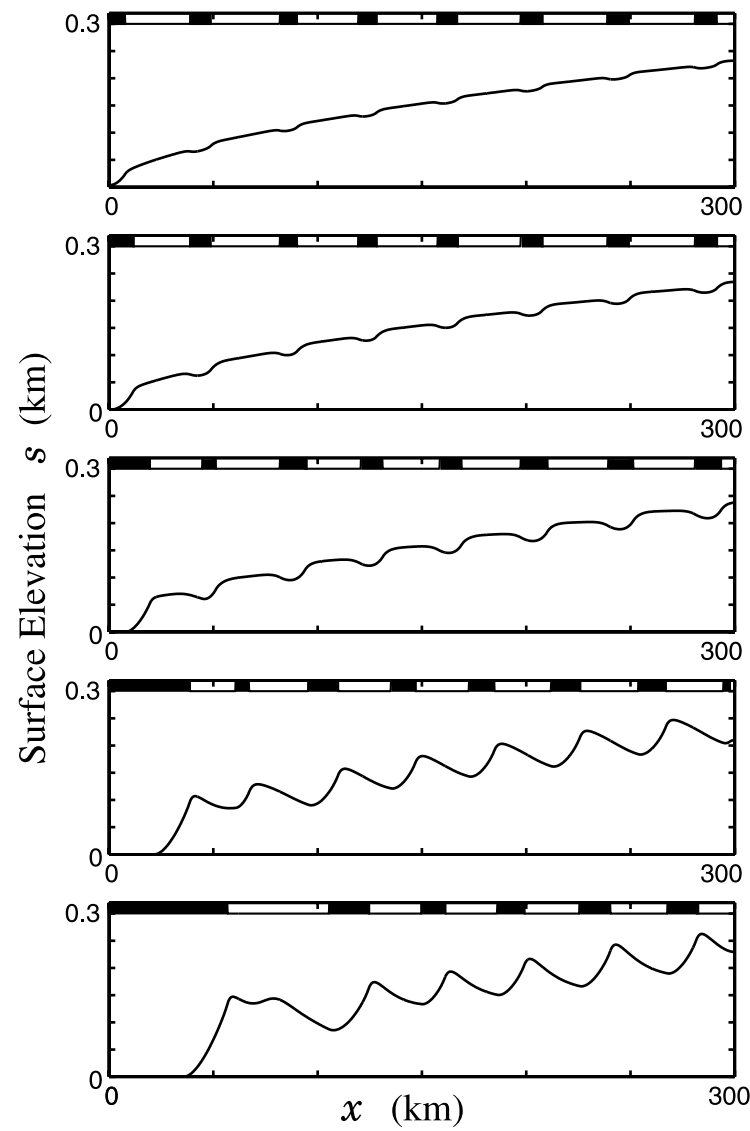

b
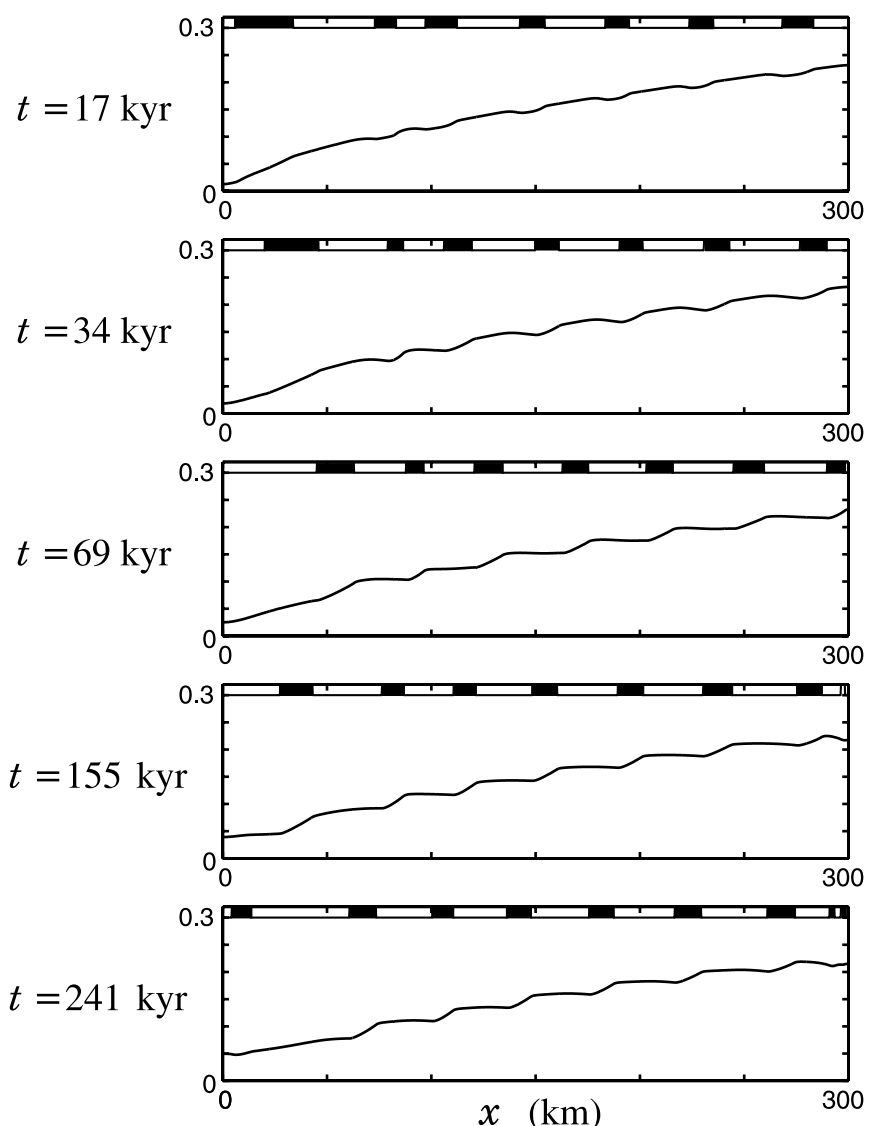

Figure 6. Topography evolution on a hypothetical polar ice cap under (a) poleward wind and (b) equatorward or katabatic wind. Black and white strips at the top of each plot show the corresponding albedo pattern in plan view.

When used with our dispersion relation, these simultaneous equations give

$$
\begin{aligned}
k_{0} & =\operatorname{Re} k_{0}+i \operatorname{Im} k_{0}=\frac{-\nu_{2}}{u}\left(1+i \sqrt{\frac{\nu_{1}}{\nu_{1}-\gamma}}\right) \quad \text { and } \\
c^{\star} & =\frac{u\left(\nu_{1}-\gamma\right)}{-\nu_{2}} .
\end{aligned}
$$

[39] Finally, the pattern wavelength, $\Lambda$, is found by conserving the number of nodes (zero crossings of $\tilde{f}$ or $\tilde{\phi}$ ) as they pass from the leading edge to the fully developed pattern [Dee and Langer, 1983]. In a reference frame moving with the envelope, the leading edge amplitude oscillates at the frequency $\left|\operatorname{Im}\left(\omega\left(k_{0}\right)-c^{*} k_{0}\right)\right| / 2 \pi$, whereas this frequency in the developed pattern is $c^{*} / \Lambda$. Thus $\Lambda=$ $2 \pi c^{*} /\left|\operatorname{Im}\left(\omega\left(k_{0}\right)-c^{*} k_{0}\right)\right|$ [Dee and Langer, 1983], which, together with equations (39) and (41), yields

$$
\Lambda=\frac{\pi u}{-\nu_{2}} \sqrt{\frac{\nu_{1}-\gamma}{\nu_{1}}} .
$$

This result implies $\Lambda \propto u$, consistent with how length is scaled in the model, but more importantly that $\Lambda$ is independent of the vapor boundary condition. Owing to this property, we could produce a first-order match of the observed albedo spacing without running simulations with different boundary conditions. For instance, with a $3 \mathrm{~m} \mathrm{~s}^{-1}$ wind and $\gamma=0.5$, equation (42) predicts $\Lambda=8$ dimensionlessly, or $80 \mathrm{~km}$ under our current $[x]$ scale of $10 \mathrm{~km}$. To match an observed $30 \mathrm{~km}$ spacing would require the moisture layer thickness $h$ (Table 1) to be changed to $40 \mathrm{~m}$, in order to reduce $[x]$ by the factor $3 / 8$ (see equation (15)). In contrast, if we wish to use the value of $h$ in Table 1, then either the wind speed or the dust stabilization rate $\gamma$ must be adjusted for the match (e.g., a $1 \mathrm{~m} \mathrm{~s}^{-1}$ wind requires $\gamma=0.2$ and a $10 \mathrm{~m} \mathrm{~s}^{-1}$ wind requires $\gamma=1.7$ ). Equation (42) identifies $\gamma$ as an extra parameter (other than the product $h u$ ) that controls the spacing. To constrain $\gamma$ will require further modeling to resolve the dust exchange processes parameterized in the present model.

\subsection{Trough Evolution}

[40] Once an albedo pattern emerges, the nonuniform sublimation rate associated with it will drive the evolution of surface topography, and slope effects become important. Equations (17), (18), and (19) then all have to be solved. We do this by taking $\epsilon=\mathrm{Pe}^{-1}=0$ as before.

[41] Figure 6 shows snapshots from two simulations of a hypothetical ice cap over a 241-kyr period, Figure 6 a with 
the wind blowing poleward and Figure $6 \mathrm{~b}$ with the wind blowing equatorward. We assume the ice to be uniformly bright initially $(f(x, 0)=0)$ and use the parameters $f_{0}=0.2$, $\gamma=1, \kappa=10,|u|=2$, and the inflow vapor pressures $\phi=1$ (Figure 6a) and $\phi=2$ (Figure 6b). These choices are by no means unique, but illustrate that troughs can form under either wind direction; we consider both directions because katabatic winds may not have prevailed at all times on the ice caps. Remember the $x$ axes here follow the wind trajectory, not a meridian.

[42] Both simulations show troughs forming and migrating toward the pole. Bistable albedo patterns develop and mature within the first kyr (in the manner described in section 6.2), with their dark regions coinciding with troughs and scarps. Trough migration rates of $\sim 0.1$ to $1 \mathrm{~m} \mathrm{yr}^{-1}$ are typical. These rates depend critically on the assumed sublimation rate factor $D$ in the model $\left(8.25 \times 10^{-5}\right)$ as well as on local surface slope. We caution that comparison of the sublimation and migration rates reported here with those inferred in other studies should bear in mind substantial uncertainties in D [Ivanov and Muhleman, 2000] (besides the fact that our model is not meant to describe ice flow accurately).

[43] It is found that deep troughs form efficiently under poleward wind (Figure 6a), whereas if the wind is reversed, a shallow trough sequence that forms early on is succeeded by a step-like topography (Figure 6b, after $34 \mathrm{kyr}$ ). The different behavior is caused by an asymmetric accublation pattern. As shown by the last three plots of Figure 5 (at $t=$ $350 \mathrm{yr}$ ), rapid sublimation occurs as dry air enters a dark region from upwind, but the sublimation rate drops as the air acquires moisture over the region. When $u>0$ (Figure 6a), this means that the lower part of each scarp sublimes faster than its upper part, which steepens the scarp wall and deepens the trough at its foot. When $u<0$ (Figure 6b), this effect flattens the scarps instead, so ice flow can more easily obliterate the troughs. We do not know whether these results explain the different character of the troughs on the north and south polar caps. But since the mass balances on the smooth terrains and on the scarps are respectively positive and negative, our simulations predict truncation of isochronous layers of ice by the migrating scarps, which is consistent with the exposure of ice-cap internal stratigraphy on their walls [e.g., Milkovich and Head, 2005].

\section{Conclusions}

[44] This paper is about placing structural constraints on a mechanism to explain polar troughs, once we accept that ice surfaces may assume bright and dark states via a feedback involving the influence of dust on surface albedo. We show how an albedo pattern in such states can develop from spatial instability, and then drive trough evolution.

[45] The mechanism requires four conditions. For the albedo feedback to work, (1) dust stabilization must be weak $\left(\gamma<v_{1}\right)$, and (2) the state of the atmosphere must be such that the polar ice surface is deflational with respect to dust $\left(f_{0}<1\right)$. For spatial patterning to work, (3) winds must advect moisture over the ice (this is not a problem on a windy Mars). For troughs to evolve, (4) ice flow must be slow enough not to close them ( $\kappa$ not being too large). The troughs and bistable albedo observed on both residual polar caps today (Figure 1c) suggest that conditions 1, 2, and 4 are met or have been met in the recent past.

[46] A consequence of condition 2 is that unconsolidated dust surfaces underlain by ice should deflate and become brighter than elsewhere on Mars if the ice has zero mass balance. In this connection, the south residual cap is bordered by layered deposits expressing a trough topography, but these deposits appear to be covered entirely by material as dark as the surroundings (compare Figure 1c with Figure 1a). If the deposits are ice-rich under the surface, then the question arises whether a large-scale albedo pattern did exist on them but was reset by a subsequent switch to inflational conditions $\left(f_{0}>1\right)$, for example, caused by climate change.

[47] Preliminary scaling shows that our wind-activated instability mechanism can match the observed spacing of troughs when atmospheric parameters $(u$ and $h)$ having sensible orders of magnitude are assumed. To constrain the match tightly, however, requires coupling our model to a sophisticated description of the atmosphere. In a mesoscale study of the north polar atmosphere, Tyler and Barnes [2005] simulated summer katabatic winds $\sim 300 \mathrm{~m}$ deep, blowing at $\sim 5 \mathrm{~m} \mathrm{~s}^{-1}$. While their wind speed supports our choice of $u$, comparing their wind-layer depth with $h$ is problematic because $h$ is a moisture-layer depth, and their model strictly assumes a dry atmosphere. A future challenge is to include realistic water vapor physics into models of the Mars atmosphere. In this regard, our equations may be viewed as providing the surface boundary conditions common to such models and ice sheet models.

[48] Because albedo patterning is the limiting step of our mechanism, we suggest that the troughs today have been preconditioned by an ancient albedo imprint; thus their planform may reflect polar conditions that differ much from the present ones. With hindsight, the work by Ivanov and Muhleman [2000] might have anticipated this possibility. In one of their simulations of parabolic polar caps, they seeded a dark patch on the ice to show how it led to the formation and survival of a trough. What we have reported is an explanation for the origin of such albedo anomaly. The notion of "albedo preconditioning" would challenge theories that propose an ongoing mechanism, not involving albedo feedback, by which troughs appear and self-organize into a spiral. However, although the spatial instability discussed above supports our hypothesis of the troughs being at right angles to surface winds, our analysis of it is restricted to the wind flow direction. The hypothesis awaits study with a two-dimensional model. In such extension, it will be important to understand the inner polar circulation and how the ice caps interact with global volatile and dust cycles.

\section{Notation}

$a_{\mathrm{D}}$ dust albedo, 0.25 (value assumed in this paper).

$a_{\mathrm{I}}$ ice albedo, 0.4 (value assumed in this paper).

$a_{0}$ equilibrium albedo, 0.37 (value assumed in this paper).

$c_{0} \quad 3.55 \times 10^{12} \mathrm{~Pa}$. 
$c_{1} \quad 6140 \mathrm{~K}$.

$D$ sublimation rate factor, $1.65 \times 10^{-5}$ to $8.25 \times 10^{-5}$ [Ivanov and Muhleman, 2000].

$D_{v} \mathrm{H}_{2} \mathrm{O}$-vapor diffusion coefficient, $\sim 10^{-3} \mathrm{~m}^{2} \mathrm{~s}^{-1}$.

$f$ surface dust fraction, $0 \leq f \leq 1$.

$f_{0}$ equilibrium dust fraction, 0.2 (value assumed in this paper).

$g_{0}$ rate of dust stabilization due to atmospheric exchange.

$h$ moisture layer thickness, $\mathrm{m}$.

$I_{0}$ solar flux, $590 \mathrm{~W} \mathrm{~m}^{-2}$.

$k$ Boltzmann constant, $1.38 \times 10^{-23} \mathrm{~J} \mathrm{~K}^{-1}$.

$L \quad \mathrm{H}_{2} \mathrm{O}$ latent heat of sublimation, $2.834 \times 10^{6} \mathrm{~J} \mathrm{~kg}^{-1}$.

$\dot{m}$ sublimation rate, $\mathrm{kg} \mathrm{m}^{-2} \mathrm{~s}^{-1}$.

$p_{s}$ saturation vapor pressure, $\mathrm{Pa}$.

Pe flow Peclet number.

$m_{w}$ water molecular mass, $0.018 \mathrm{~kg} \mathrm{~mol}^{-1} \div 6.02 \times 10^{23}$ $\mathrm{mol}^{-1}$.

$s$ ice surface elevation, $\mathrm{m}$.

$T_{0}$ moisture layer temperature, $\mathrm{K}$.

$u$ wind velocity, $\mathrm{m} \mathrm{s}^{-1}$.

$x$ distance along wind trajectory, $\mathrm{m}$.

$t$ time, s.

$\Delta$ characteristic ice thickness (section 3.3), m.

$\phi \quad \mathrm{H}_{2} \mathrm{O}$ vapor pressure, $\mathrm{Pa}$.

$\rho_{i} \quad$ ice density, $900 \mathrm{~kg} \mathrm{~m}^{-3}$

$\sigma \quad$ Stefan-Boltzmann constant, $5.67 \times 10^{-8} \mathrm{~W} \mathrm{~m}^{-2} \mathrm{~K}^{-4}$.

$\theta_{0}$ reference zenith angle, $72.5^{\circ}$ (value assumed in this paper).

[49] Acknowledgments. This paper benefited from stimulating discussions with Shane Byrne, who helped us also to produce Figures 1a and 1b. We thank Chris Clark for comments on the manuscript, and Sarah Milkovich and two anonymous persons for providing critical reviews. Felix $\mathrm{Ng}$ acknowledges the support of a Leavitt Research Fellowship at the Department of EAPS at MIT and the wonderful support of colleagues there while this work was undertaken. Additional support was provided by the NASA Mars Data Analysis Program.

\section{References}

Bass, D. S., and D. A. Paige (2000), Variability of Mars' north polar water ice cap: II. Analysis of Viking IRTM and MAWD data, Icarus, 144, $397-409$.

Bass, D. S., K. E. Herkenhoff, and D. A. Paige (2000), Variability of Mars' north polar water ice cap: I. Analysis of Mariner 9 and Viking Orbiter Imaging data, Icarus, 144, 382-396.

Bibring, J.-P., et al. (2004), Perennial water ice identified in the south polar cap of Mars, Nature, 428, 627-630.

Blasius, K. R., J. A. Cutts, and A. D. Howard (1982), Topography and stratigraphy of Martian polar layered deposits, Icarus, 50, $140-$ 160.

Byrne, S., and A. P. Ingersoll (2003), A sublimation model for Martian south polar ice features, Science, 299, 1051-1053.

Byrne, S., and A. B. Ivanov (2004), Internal structure of the Martian south polar layered deposits, J. Geophys. Res., 109, E11001, doi:10.1029/ 2004JE002267.

Calvin, W. M., and T. N. Titus (2004), Summer season variability of the north residual cap of Mars from MGS-TES, in Lunar and Planetary Science Conference XXXV [CD-ROM], Abstract 1455, Lunar and Planet. Inst., Houston, Tex. (Available at http://www.lpi.usra.edu/publications/ abstracts.shtml)

Cutts, J. A. (1973), Wind erosion in the Martian polar regions, J. Geophys. Res., 78(20), 4211-4221.

Dee, G., and J. S. Langer (1983), Propagating pattern selection, Phys. Rev. Lett., 50(6), 383-386.

Dee, G. T., and W. van Saarloos (1988), Bistable systems with propagating fronts leading to pattern formation, Phys. Rev. Lett., 60(25), $2641-2644$

Durham, W. B., S. H. Kirby, L. A. Stern, and S. C. Circone (2003), The rheology of $\mathrm{CO}_{2}$ clathrate hydrate and other candidate ices in the Martian polar caps, in 3rd International Conference on Mars Polar Science and Exploration [CD-ROM], Abstract 8132, Lunar and Planet. Inst., Houston, Tex. (Available at http://www.lpi.usra.edu/meetings/polar2003/ abstractsess.html)

Farmer, C. B., D. W. Davies, and D. D. LaPorte (1976), Mars: Northern summer ice cap-Water vapor observations from Viking 2, Science, 194, $1339-1341$.

Fisher, D. A. (1993), If Martian ice caps flow: Ablation mechanisms and appearance, Icarus, 105, 501-511.

Fisher, D. A. (2000), Internal layers in an 'accublation' ice cap: A test for flow, Icarus, 144, 289-294.

Herkenhoff, K. E., and J. J. Plaut (2000), Surface ages and resurfacing rates of the polar layered deposits on Mars, Icarus, 144, 243253 .

Houghton, J. T. (1986), The Physics of Atmospheres, 2nd ed., Cambridge Univ. Press, New York.

Howard, A. D. (1978), Origin of the stepped topography of the Martian poles, Icarus, 34, 581-599.

Howard, A. D. (2000), The role of eolian processes in forming surface features of the Martian polar layered deposits, Icarus, 144, $267-288$.

Howard, A. D., J. A. Cutts, and K. R. Blasius (1982), Stratigraphic relationships within Martian polar cap deposits, Icarus, 50, 161215.

Hvidberg, C. S. (2003), Relationship between topography and flow in the north polar cap on Mars, Ann. Glaciol., 37, 363-369.

Ivanov, A. B., and D. O. Muhleman (2000), The role of sublimation for the formation of the northern ice cap: Results from the Mars Orbiter Laser Altimeter, Icarus, 144, 436-448.

Jakosky, B. M., and R. J. Phillips (2001), Mars' volatile and climate history, Nature, 412, 237-244.

James, P. B., and B. A. Cantor (2001), Martian north polar cap recession: 2000 Mars Orbiter Camera observations, Icarus, 154, $131-144$.

Kieffer, H. H. (1990), $\mathrm{H}_{2} \mathrm{O}$ grain size and the amount of dust in Mars' residual north polar cap, J. Geophys. Res., 95(B2), 14811493.

Kieffer, H. H., S. C. Chase Jr., T. Z. Martin, E. D. Miner, and F. D. Palluconi (1976), Martian north pole summer temperatures: Dirty water ice, Science, 194, 1341-1344.

Kolb, E. J., and K. L. Tanaka (2001), Geologic history of the polar regions of Mars based on Mars Global Surveyor data: II. Amazonian Period, Icarus, $154,22-39$.

Langevin, Y., F. Poulet, J.-P. Bibring, B. Schmitt, S. Douté, and B. Gondet (2005), Summer evolution of the north polar cap of Mars as observed by OMEGA/Mars Express, Science, 307, 1581-1584.

Laskar, J., B. Levrard, and J. F. Mustard (2002), Orbital forcing of the Martian polar layered deposits, Nature, 419, 375-377.

Milkovich, S. M., and J. W. Head III (2005), North polar cap of Mars: Polar layered deposit characterization and identification of a fundamental climate signal, J. Geophys. Res., 110, E01005, doi:10.1029/ 2004JE002349.

Murray, J. D. (2002), Mathematical Biology: I. An Introduction, 3rd ed., Springer, New York.

Ng, F. S. L., and M. T. Zuber (2003), Albedo feedback in the patterning mechanisms of Martian polar caps, in 3rd International Conference on Mars Polar Science and Exploration [CD-ROM], Abstract 8061, Lunar and Planet. Inst., Houston, Tex. (Available at http://www.lpi.usra.edu/ meetings/polar2003/abstractsess.html)

Paterson, W. S. B. (1994), The Physics of Glaciers, 3rd ed., Elsevier, New York.

Pelletier, J. D. (2004), How do spiral troughs form on Mars?, Geology, 32(4), 365-367.

Rovinsky, A. B., and M. Menzinger (1992), Chemical instability induced by a differential flow, Phys. Rev. Lett., 69(8), $1193-$ 1196.

Rovinsky, A. B., S. Nakata, V. Z. Yakhnin, and M. Menzinger (1996), Control of activator-inhibitor systems by differential transport, Phys. Lett. A, 216, 262-268.

Satnoianu, R. A., and M. Menzinger (2000), Non-Turing stationary patterns in flow-distributed oscillators with general diffusion and flow rates, Phys. Rev. E, 62(1), 113-119.

Smith, D. E., et al. (1998), Topography of the Northern Hemisphere of Mars from the Mars Orbiter Laser Altimeter, Science, 279, 16861692.

Smith, D. E., et al. (2001a), Mars Orbiter Laser Altimeter: Experiment summary after the first year of global mapping of Mars, J. Geophys. Res., 106(E10), 23,689-23,722.

Smith, D. E., M. T. Zuber, and G. A. Neumann (2001b), Seasonal variations of snow depth on Mars, Science, 294, 2141-2146. 
Thomas, P., S. Squyres, K. Herkenhoff, A. Howard, and B. Murray (1992), Polar Deposits of Mars, in Mars, edited by H. H. Kieffer et al., pp. 767795, Univ. of Ariz. Press, Tucson.

Turing, A. M. (1952), The chemical basis of morphogenesis, Philos. Trans R. Soc. London, Ser. B, 237, 37-72.

Tyler, D., Jr., and J. R. Barnes (2005), A mesoscale model study of summertime atmospheric circulations in the north polar region of Mars, J. Geophys. Res., 110, E06007, doi:10.1029/2004JE002356.

van Saarloos, W. (1987), Dynamical velocity selection: Marginal stability, Phys. Rev. Lett., 58(24), 2571-2574.

Walgraef, D. (1997), Spatio-Temporal Pattern Formation, Springer, New York.
Zuber, M. T., et al. (1998), Observations of the north polar region of Mars from the Mars Orbiter Laser Altimeter, Science, 282, 20532060

F. S. L. Ng, Department of Geography, University of Sheffield, Winter Street, Sheffield S10 2TN, UK. (f.ng@sheffield.ac.uk)

M. T. Zuber, Department of Earth, Atmospheric and Planetary Sciences, Massachusetts Institute of Technology, 77 Massachusetts Avenue, Cambridge, MA 02139, USA. (zuber@mit.edu) 